Journal of Computer Science (Special Issue): 77-79, 2005

ISSN 1549-3636

(C) 2005 Science Publications

\title{
Constructing Longest Lifetime Route in Mobile Ad-hoc Networks
}

\author{
${ }^{1}$ Fredrick Mtenzi and ${ }^{2}$ Yingyu Wan \\ ${ }^{1}$ Computer Science Department, School of Computing, Dublin Institute of Technology \\ Kevin Street, Dublin 8, Ireland \\ ${ }^{2}$ Department of Computer Science, University of Science and Technology of China \\ Hefei 230027, P.R.China
}

\begin{abstract}
A mobile ad hoc network (MANET) is an autonomous system consisting of mobile hosts connected by wireless links. Every host can move in any direction at any speed and time. This leads to a dynamic topology as hosts move constantly. MANET broadcast messages to each hosts, the transmission of one host can be heard by all hosts in its communication range. If two hosts are not located in each other's transmission range, intermediate relay hosts must be employed as bridges to build communication paths. This is the multihop characteristic of the mobile ad hoc network, for which routing decisions must be made for far-away hosts to communicate. Node mobility causes links between nodes to break frequently, thus terminating the lifetime of the routes containing those links. An alternative route has to be discovered once a link is detected as broken, incurring extra route discovery overhead and packet latency. Traditionally route discovery has been done using flooding based approaches, which sometimes leads to broadcast storm problem. In this paper, we study the problem of how to construct a route with the longest lifetime for any given one-to-one communication request as a solution to link breakage in MANET. An algorithm is proposed with time complexity of $O(m+n \log n)$, where $n$ is the number of the nodes and $m$ is the number of the links. The proposed algorithm complexity is similar to that of the Dijkstra algorithm implemented using Fibonacci heap.
\end{abstract}

Key words: MANET, links, longest lifetime routing

\section{INTRODUCTION}

A mobile ad hoc network (MANET) is an autonomous system consisting of mobile hosts connected by wireless links. It can be flexibly, quickly deployed and dismantled easily for many practical applications such as battlefield operations, festival grounds, search and rescue and disaster relief emergency. Unlike wired networks or cellular networks, there is no physical infrastructure and central administration in mobile ad hoc networks. Every host can move in any direction at any speed and any time. These factors among others introduce a dynamic topology. Due to the broadcast advantage of wireless communication, the transmission of one host can be heard by all hosts in its communication range. If two hosts are not located in each other's transmission range, intermediate relay hosts must be employed as bridges to build communication paths. This is the multihop characteristic of the mobile ad hoc network, for which routing decisions must be made for far-away hosts to communicate. When choosing a routing path among several hosts, there are usually many factors to be considered, such as route length, route quality, signal strength, path bandwidth and route lifetime. Mobile ad hoc network hosts are usually light-weight and batterypowered. Compared to wired lines, wireless links have much less available bandwidth.
Routing protocols used in mobile ad hoc networks can be divided into two categories: table-driven (proactive) and on-demand (reactive). Examples of table-driven protocols include optimized link state routing $(\mathrm{OLSR})^{[1]}$ and destination-sequenced distance vector $(\mathrm{DSDV})^{[2]}$. These protocols require nodes to maintain a route table for all other nodes so that a route is always available when a packet is ready to be transmitted. However, on-demand protocols attract more interest than table-driven protocols because they only initiate a route discovery process when a packet is ready to be transmitted. Without the necessity of persistent maintenance of a routing table, where shortest path algorithms are usually applied, on-demand protocols typically have lower routing overhead than table-driven protocols. Examples of on-demand protocols include dynamic source routing (DSR) ${ }^{[3]}$ and ad-hoc on-demand distance vector $(\mathrm{AODV})^{[4]}$.

Node mobility is one of the most important characteristics that affect the performance of mobile ad hoc networks. When a link breaks due to node mobility, the routes containing this link also become invalid. Therefore, an alternative route has to be discovered. For example, in on-demand routing protocols, a route discovery process will be invoked to search for a new route, which is intrinsically flooding. Flooding suffers from the notorious broadcast storm problem $^{[5]}$ and may result in excessive redundancy,

Corresponding Author: $\quad$ Fredrick Mtenzi, Computer Science Department, School of Computing, Dublin Institute of Technology, Kevin Street, Dublin 8, Ireland, Tel: +353-1-4024954, Fax: +353-1-4024985 
contention and collision, which will cause high protocol overload and interference to ongoing traffic. This issue becomes more serious when the mobility of the network is high. Several schemes such as probabilistic, counterbased, distance-based, location-based and cluster-based have been proposed to alleviate this problem ${ }^{[5]}$. These schemes even the adaptive approaches help in relieving the broadcast storm problem in MANET, they do not eliminate it completely ${ }^{[6,7]}$.

In order to minimize the adverse impact of link breakage and corresponding route discoveries from node mobility, an intuitive approach is to find routes with longest lifetime. So if a route with long lifetime is selected, the frequency of route discovery process being invoked will be reduced and the protocol overload will also be reduced. This is so because most of existing protocols attempt to find a route with shortest distance (such as $\mathrm{DSR}^{[3]}$ ) or one with minimum energy consumption $^{[8,9]}$. Few considers the lifetime of the route ${ }^{\text {[Error! Reference source not found.0-13] }}$. We define lifetime of a route as the time duration starting from beginning of broadcast service until the first node in the route fails due to energy exhaustion or link breakage.

\section{LONGEST LIFETIME CONSTRUCTION}

We assume that at the beginning, a snapshot of the network is given. From the snapshot we know which links exist and the lifetime of each, which means how long the link can be maintained before it is disconnected. The lifetime of links can be obtained by a prediction of each node signal strength and its movement ${ }^{\text {[Error! Reference source not found.0] }}$. Thus the network can be modeled as a graph $G=(V, E)$ with a weight function $l: E \rightarrow R^{+}$, where $E$ is the set of links, $l(e)$ is the lifetime of link $e \in E$. An example is shown in Fig. 1 , where in (a) the arrow beside each node indicates the movement of the node and in (b) the number beside each link represents the lifetime of the link.

Definition 1: Longest lifetime routing I (LLR-I): Given a graph $G=(V, E, l)$ and a communication request $(s, t)$, the problem is to find a path $p$ from $s$ to $t$ such that the lifetime of $p$ is the longest. The lifetime of $p$ is defined as $\min _{e \in p}\{l(e)\}$.

Let $P$ be the set of all paths from $s$ to $t$, the objective of LLR-I is to find $\max _{p \in P} \min _{e \in p}\{l(e)\}$, which is in fact the same as the traditional bottleneck path problem. The problem can be solved by Dijkstra algorithm with minor modification.

Note that during communication, there is always a delay on transmitting node, including the waiting time in queue and the transmitting time. Taking into consideration all these factors, the definition of the lifetime of a path can be defined as follows:
Definition 2: Longest Lifetime Routing II (LLR-II): Given a graph $\mathrm{G}=(\mathrm{V}, \mathrm{E}, \mathrm{l})$ and a communication request $(s, t)$, let $\Delta$ be the delay on each node,

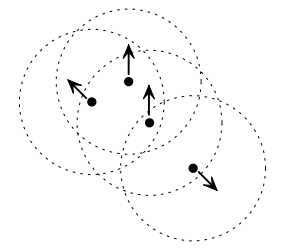

(a)

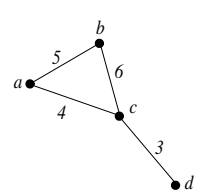

(b)
Fig. 1: Model mobile ad hoc networks as a weighted graph

the problem is to find a path $p$ from $s$ to $t$ such that the lifetime of $p$ is the longest. The lifetime of $p$ is defined as $l_{p}=\min _{e \in p}\left\{l(e)-d_{p}(e) \Delta\right\}$, where $d_{p}(e)$ is the sequence number of $e$ in $p$ in the order from s to $t$.

For those problems which can be solved by Dijkstra-like algorithm, they must satisfy the following optimal substructure property: there exists an optimal path $S P(s, t)$, if $k$ is an intermediate node in $\operatorname{SP}(s, t)$, then the $S P^{\prime}(s, k)$ is an optimal path from $s$ to $k$, where $S P^{\prime}(s, k)$ is the subpath from $s$ to $k$ in $S P(s, t)$. Unfortunately, for LLR-II, this property doesn't hold. For example, in Fig. 1(b) let $\Delta=1$, then the longest lifetime path from $a$ to $c$ is $a \rightarrow b \rightarrow c$, the lifetime is $\min \{5-1,6-2\}=4$, while the longest lifetime path from $a$ to $d$ is $a \rightarrow c \rightarrow d$, the lifetime is $\min \{4-1,3-2\}=1$.

We find that LLR-II has the following property, which is similar to the optimal substructure property of Dijkstra algorithm.

Theorem 1: For any $u, v \in V$, let $P(u, v)$ denote $a$ longest lifetime path from $u$ to $v$. If $k$ is an intermediate node in $P(s, t)$, then the path $s \stackrel{P(s, t)}{\longrightarrow} k \stackrel{P(k, t)}{\longrightarrow} t$ is also a longest lifetime path from s to $t$.

Proof: Let $P^{\prime}(s, k)$ and $P^{\prime}(k, t)$ denote $s \stackrel{P(s, t)}{\longrightarrow} k$ and $k \stackrel{P(s, t)}{\longrightarrow} t$ respectively. And let $P^{\prime \prime}(s, t)$ denote the path $s \stackrel{P(s, t)}{\longrightarrow} k \stackrel{P(k, t)}{\longrightarrow} t$, which is concatenated by $P^{\prime}(s, k)$ and $P(k, t)$. Let $h$ be the length of $P^{\prime}(s, k)$.

Then $l_{p^{\prime}(k, t)} \leq l_{p(k, t)}$ and

$$
\begin{aligned}
l_{P(s, t)} & =\min _{e \in P(s, t)}\left\{l(e)-d_{P(s, t)}(e) \Delta\right\} \\
& =\min _{\min _{e \in P^{\prime}(s, k)}\left\{l(e)-d_{P^{\prime}(s, k)}(e) \Delta\right\},} \\
& \left.\min _{e \in P^{\prime}(k, t)}\left\{l(e)-d_{P^{\prime}(k, t)}(e) \Delta\right\}-h \Delta\right\} \\
& =\min \left\{l_{P^{\prime}(s, k)}, l_{P^{\prime}(k, t)}-h \Delta\right\}
\end{aligned}
$$




$$
\begin{aligned}
& \leq \min \left\{l_{P^{\prime}(s, k)}, l_{P(k, t)}-h \Delta\right\} \\
& =l_{P^{\prime \prime}(s, t)}
\end{aligned}
$$

Since $P(s, t)$ is a longest lifetime path, so $l_{P(s, t)}=l_{P^{\prime \prime}(s, t)}$ and $P^{\prime \prime}(s, t)$ is also a longest lifetime path from $s$ to $t$.

We design an algorithm for LLR-II

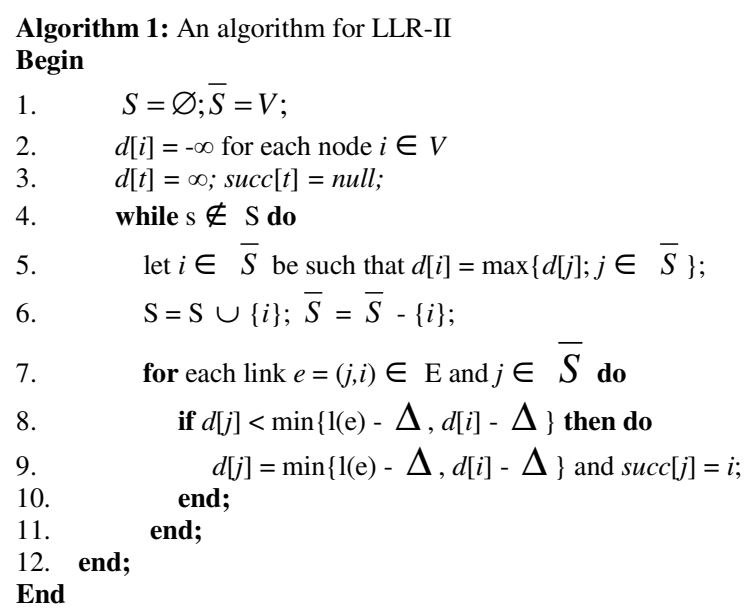

Theorem 2: The path found in algorithm 1 is a longest lifetime path from $s$ to $t$. The complexity of it is $O(m+n \log n)$ where $m=|E|$ and $n=|V|$.

Proof: The correctness of algorithm 1 follows theorem 1. Note that the algorithm is much similar to Dijkstra algorithm. They have the same time complexity. An implementation based on Fibonacci heap ${ }^{[14]}$ runs in time $O(m+n \log n)$.

\section{CONCLUSION}

As mobile ad hoc networks are increasing in popularity and being deployed in a lot of applications, the need to improve their performance and finally achieving a much higher quality of service is becoming evident. A lot of research is being undertaken to improve MANET. In this study, we discuss and consider the longest lifetime construction problem and propose an algorithm for it. The algorithm proposed achieves a time complexity of $O(m+n \log n)$, which is similar to that of the Dijkstra algorithm implemented using Fibonacci heap.

It is worthy pointing out that, longest lifetime routes are more stable than shortest path routes in terms of packet delivery ratio and packet latency. Using the longest lifetime route, packets can be delivered within certain delay constraints and without any packet loss as long as there is no congestion along the path. In contrast, relatively frequent link breakage from shortest path routes may cause packet drops and may jitter the packet delay. Using longest lifetime route less frequent route maintenance is required, which is usually a costly flooding based procedure. However, some of the drawbacks of longest lifetime route include more energy being consumed for delivering the same number of packets as there are more hops along the route and self interference by successive nodes in a route which may make them not as attractive as shorter routes.

We currently working on the design of a distributed algorithm and expect to evaluate its performance experimental. Further, we expect to consider a number of other factors at the same time, such as the energy consumption and signal strength.

\section{REFERENCES}

1. Laouiti, A., A. Qayyum, L. Viennot, P. Jacquet and T. Clausen, 2001. Optimized link state routing protocol.

2. Charles, E.P. and P. Bhagwat, 1994. Highly dynamic destination-sequenced distance-vector routing (DSDV) for mobile computers. In SIGCOMM, pp: 234-244.

3. David, B.J. and D.A. Maltz, 1996. Dynamic Source Routing in Ad Hoc Wireless Networks. In Imielinski and Korth, eds., Mobile Computing, Vol. 353 of The Kluwer International Series in Engineering and Computer Science, Kluwer Academic Publishers.

4. Charles, E.P. and E.M. Belding-Royer, 1999. Adhoc on-demand distance vector routing. In 2nd Workshop on Mobile Computing Systems and Applications (WMCSA '99), pp: 90-100.

5. Sze-Yao, N., Y.-C. Tseng, Y.-S. Chen and J.-P. Sheu, 1999. The broadcast storm problem in a mobile ad hoc network. In Proc. MOBICOM, pp: 151-162.

6. Chih, S.H. and Y.C. Tseng, 2002. An efficient reliable broadcasting protocol.

7. Ni. S.Y. and Y.C. Tseng, 2002. Adaptive approaches to relieving broadcast storms.

8. Singh, S., M. Woo and C.S. Raghavendra, 1998. Power-aware routing in mobile ad hoc networks. In MOBICOM, pp: 181-190.

9. Wei, X., F. Mtenzi, B. Wu and Y. Wan, 2003. An energy efficient routing algorithm with priority for mobile ad hoc networks (pdsr). In Lambertus Hesselink, editor, Wireless and Optical Communications WOC 2003, The International Association of Science and Technology for Development (IASTED), ACTA Press.

10. Agarwal, S., A. Ahuja, J.P. Singh and R. Shorey, 2000. Route-lifetime assessment based routing (RABR) protocol for mobile ad-hoc networks. In IEEE Intl. Conf. on Communications, 3: 1697-1701.

11. Cheng, Z. and W.B. Heinzelman, 2004. Exploring long lifetime routing (LLR) in ad hoc networks. In Proc. 7th Intl. Symp. on Modeling Analysis and Simulation of Wireless and Mobile Systems ( MSWiM), pp: 203-210.

12. Sankar, A. and Z. Liu, 2004. Maximum lifetime routing in wireless ad-hoc networks. In INFOCOM.

13. Wang, Y., M. Martonosi and L.-S. Peh, 2004. Mario: Mobility-adaptive routing using route lifetime abstractions in mobile ad hoc networks. SIGMOBILE Mobile Computing and Communications Rev., 8: 77-81.

14. Cormen, T., C. Leiserson and R. Rivest, 1990. Introduction to Algorithms, MIT Press. 